Results: 42 participants ( 26 family carers and 16 care home staff) took part in the baseline interviews, and 20 purposefully sampled participants (11 family carers and 9 care home staff) were followed up. Prior to vaccination roll out in the UK, at baseline, family carers expressed concern about a lack of clear guidance throughout the pandemic, with care homes delivering care differently and disparities noted in the levels and types of visiting allowed for family members. Lack of communication between care homes and family members, but also government and care homes, led to family carers feeling excluded and concerned about the well-being of their relative. Data on follow-up interviews are still being analysed.

Conclusions: This is the first empirical evidence to show how the pandemic has caused severe difficulties in providing adequate care for care home residents, with not only residents, but also care home staff and family carers being negatively affected. Follow-up data will shed light onto the impact of vaccination and eased visitation rights put in place since March 2021 on care delivery and connections between family carers and residents.

\title{
211 - Changes to post-diagnostic dementia support in England and Wales during the COVID-19 pandemic
}

Alison Wheatley, Marie Poole, Louise Robinson

Background: The COVID-19 pandemic precipitated widespread change across health and social care in England and Wales. A series of lockdowns and UK Government guidance designed to reduce the spread of COVID-19 which emphasised social distancing and increased use of personal protective equipment led to changes such as increased use of remote consultation technologies and the closure of services deemed non-essential. This included many services for people with dementia and their families, such as day centres and dementia cafes.

Objective: To explore the changes made to services during the pandemic and the impact of these changes on the delivery of good post-diagnostic dementia support.

Method: Professionals who had previously been recruited to the ongoing PriDem qualitative study were approached for follow up interview. Eighteen interviews with a total of 21 professionals working in health, social care and the third sector were conducted using telephone or video conferencing. Interviews were audio recorded, transcribed and checked prior to thematic analysis.

Results: Key themes emerging from preliminary analysis of the data include: uncertainty about the future and the need to adapt quickly to shifting guidance; changing job roles and ways of working; the emotional and physical impact of the pandemic on staff working with people with dementia and their families; and the impact of changes made (e.g. increased PPE, remote working) on the ability to deliver post-diagnostic support. However, there were also some unintended positive outcomes of the changes. These included the ability to include family members living at a distance in remote consultations, allowing for more robust history-taking, as well as the uptake of technology to facilitate cross-sector and multidisciplinary working between professionals.

Conclusion: Delivering post-diagnostic dementia support during COVID-19 was challenging and forced dementia services to make adaptations. Participants expected that some of these changes would be incorporated into post-pandemic work, for example increased use of technology for multidisciplinary team meetings or blended approaches to patient-facing services involving both virtual and face to face work as appropriate. However, most participants agreed that it was not appropriate nor desirable to provide fully remote post-diagnostic support on a full time basis. 